\title{
Performance, Operational Aspects and Impact on Physics Results of the AMS Tracker.
}

\author{
A. Oliva* for the AMS-02 Tracker Collaboration. \\ *CIEMAT, Avenida Complutense 40, Madrid, Spain. \\ silicon E-mail: alberto.oliva@ciemat.es
}

\begin{abstract}
AMS-02 is a magnetic spectrometer particle detector operating onboard the International Space Station (ISS) since May 2011. Its main scientific objectives are the research of Dark Matter annihilation byproducts, direct search of primordial Anti-Matter and the precise measurement of the Cosmic-Rays (CRs) spectra. The AMS silicon Tracker is composed by 2284 double-sided silicon sensors $\left(74 \times 41 \mathrm{~mm}^{2}, 300 \mu \mathrm{m}\right.$ thick) arranged over 9 detection layers. Seven layers are placed inside the permanent magnet volume $(\mathrm{T} \sim 0.14 \mathrm{~T}$ ), while 2 layers are placed at the edges of AMS. To ensure optimal Tracker performances the Tracker Thermal Control System (TTCS), a two phases $\mathrm{CO}_{2}$ cooling system, keeps the Tracker front-end electronics temperatures stable within $1^{\circ} \mathrm{K}$. The typical spatial resolution of $10(5) \mu \mathrm{m}$ for $Z=1(Z=2)$ particles and the maximum lever arm of approximately $3 \mathrm{~m}$ give a Maximum Detectable Rigidity (MDR), i.e. the rigidity $(R=p / Z e)$ value for which $\Delta \mathrm{R} / \mathrm{R}=100 \%$, of 2 (3.2) TV. The combination of multiple measurements of the energy deposition in the silicon sensors along the trajectory, and the high-dynamic range readout electronics give a resolution on the absolute charge magnitude measurement of about $0.06(0.15)$ charge units for $Z=1(Z=6)$, with separation capabilities ranging at least up to $Z=28$. In this contribution we will give a description of the AMS Tracker operational aspects in terms of stability of the electronics performance as well as an overview on the calibrations needed to ensure the expected momentum resolution (alignment of external and internal planes) and charge discrimination (equalization of the chip responses). An example on the impact of the Tracker measurements in the analysis of the CRs chemical composition studies will be shown.
\end{abstract}

22nd International Workshop on Vertex Detectors,

15-20 September 2013

Lake Starnberg, Germany 


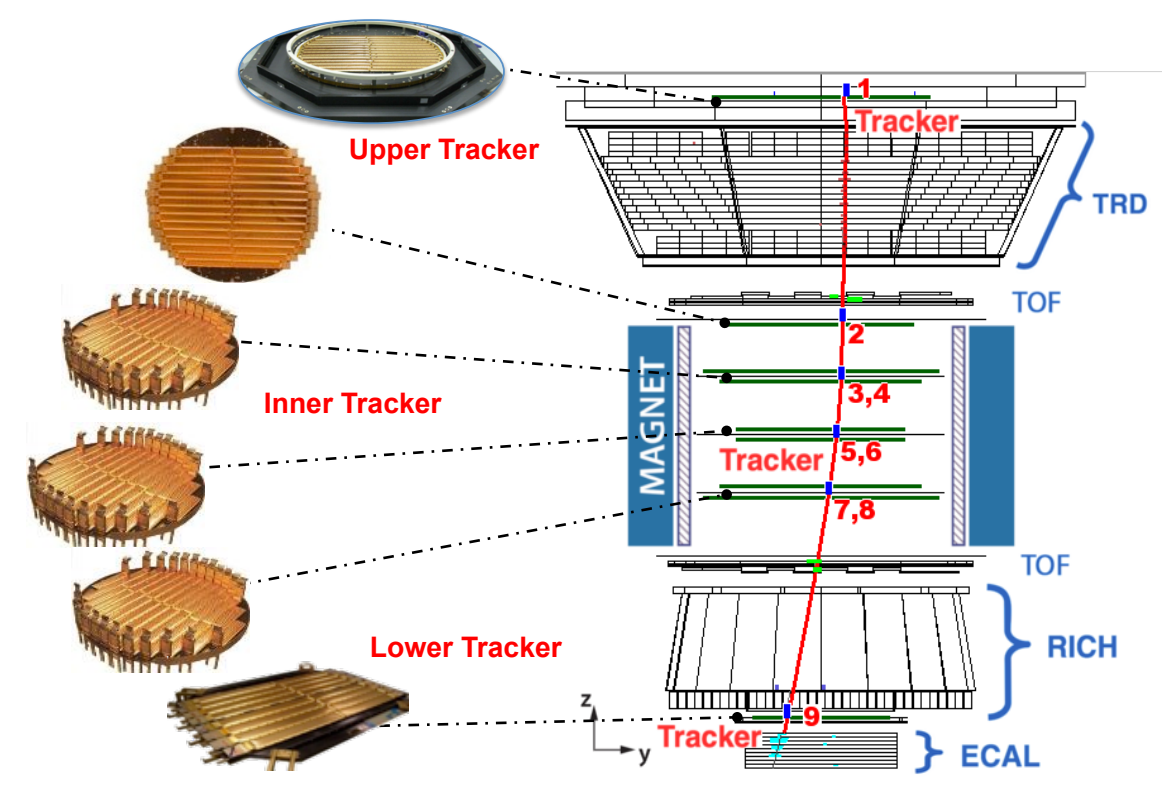

Figure 1: On the right a schematic view of AMS-02 detector in the bending plane ( $y z$ plane) is shown as well as an example of the signal released by a low energy proton (red line). On the left an exploded view of the AMS-02 Tracker is presented.

\section{The AMS-02 Detector}

The Alpha Magnetic Spectrometer (AMS) is a high-energy physics experiment built to operate in space. The prototype of the AMS detector was AMS-01, flown in 1998 on-board of the Space Shuttle Discovery in mission STS-91. A description of the instrument as well as few of the most important results of AMS-01 can be found in reference [1]. AMS-02 has been constructed improving the AMS-01 design in terms of extension of the energetic range, enlargement of the geometric acceptance and improvement of the particle identification capabilities. AMS-02 has been carried on the ISS on May 2011 by the space shuttle Endeavour in mission STS-134. The apparatus is operating since then, and is expected to operate for 20 years.

The layout of the AMS-02 detector is shown in Figure 1. It consists of 9 layers of silicon Tracker, a Transition Radiation Detector (TRD), four planes of Time-of-Flight counters (TOF), a permanent magnet, a cylindric array of Anti-Coincidence Counters (ACC) surrounding the Tracker, a Ring Imaging Čerenkov detector (RICH), and an Electromagnetic Calorimeter (ECAL) [2] [3].

The silicon Tracker detects the curvature of the charged particles bent by the $0.14 \mathrm{~T}$ magnetic field, by means of the measurement of the impact coordinates on the 9 detection layers arranged over an extension of about $3 \mathrm{~m}$. From the curvature the particle rigidity and its sign are derived. The MDR is estimated to be 2 (3.2) TV for $Z=1(Z=2)$ particles [4]. TOF evaluates the particle speed by the difference in time between the upper and lower layers, with a typical resolution of $\Delta \beta=0.04$ for $Z=1$ relativistic particles [5]. Another more precise measurement of velocity is performed by the RICH by the measurement Cerenkov radiation cone aperture with a resolution of $\Delta \beta=10^{-3}$ for $Z=1$ relativistic particles. TRD and ECAL systems are specialized detectors 


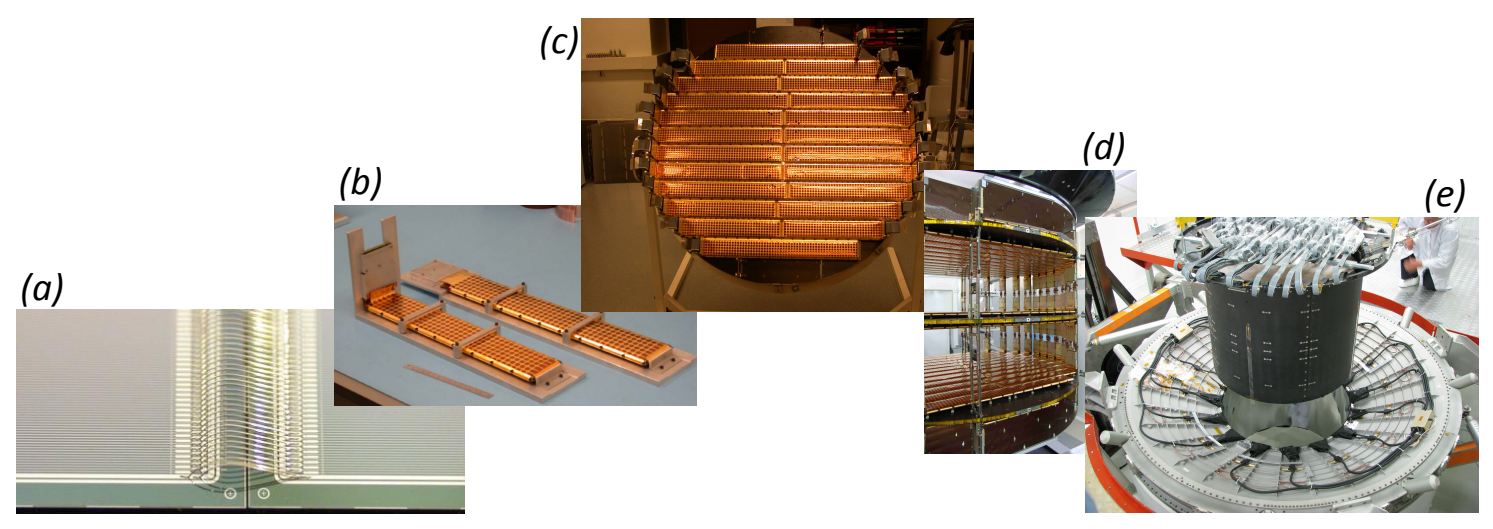

Figure 2: Various phases of the AMS-02 Tracker construction: (a) metallic bonds between silicon sensors; (b) two complete ladders, each one is composed by sensors, readout electronics and shielding; (c) a set of ladders fixed on top of the honeycomb aluminum structure constitute a Tracker layer; (d) the Inner Tracker assembly; (e) integration of the Inner Tracker into AMS-02.

designed to reach a high capabilities in electron/proton separation [6]. All the 5 sub-systems have capabilities in the absolute charge magnitude (Z) determination [5] [7] [8] [9] [10].

\section{The Silicon Tracker}

The Tracker system is composed of $2284,300 \mu \mathrm{m}$ thick n-doped double-sided silicon microstrip sensors with surface dimensions of $72 \times 41 \mathrm{~mm}^{2}$. Metallic strips are implanted over the two opposite faces of the sensor along orthogonal directions allowing the simultaneous measurement of two coordinates. On the $y$-side, along the bending coordinate, the implantation (read-out) pitch is of $27.5(110) \mu \mathrm{m}$. On the opposite side along the $x$-axis, i.e. the non-bending coordinate, strips are arranged with a larger implantation (readout) pitch of 104 (208) $\mu \mathrm{m}$.

From 9 to 15 sensors are assembled together and coupled to the Tracker Front-end Electronics (TFE) in basic functional elements called ladder for a total number of 192 ladders. Sensors are assembled along the bending coordinate, i.e. strips along $y$-axis are daisy-chained together through metallic bonds, as depicted in (a) of Figure 2, and are connected to the front-end electronics (TFE). On the other side strips of alternating sensors are connected together via an Upilex cable, giving an ambiguity in the hit positions along the coordinate $x$. The ambiguity has the periodicity of two sensor $(8.2 \mathrm{~cm})$ and is resolved during track reconstruction or by the use of the reconstructed track in combination with information coming from other sub-detectors (such as TRD or TOF) [4]. For each ladder there are 640 and 384 readout channels along $y$ and $x$ coordinates respectively, routed via $700 \mathrm{pF}$ capacitors to the TFE. The ladder is completed by supporting structures composed by thin framed layers of foam, carbon and aluminum, and an electromagnetic shield made by an Upilex foil [11]. Examples of complete ladders are presented in (b) of Figure 2.

On the TFE sits the signal amplification chain. The 1024 readout channels are processed by 64 channel, high dynamic range (up to 100 minimum-ionizing-particle signal), low consumption $(0.5 \mathrm{~mW} / \mathrm{channel}) \mathrm{VA}$ chips. A total of 6 and $10 \mathrm{VA}$ chips are used to read the signals from the $x$ and $y$ side respectively. Each VA readout channel consists of a charge sensitive amplifier, a CR- 


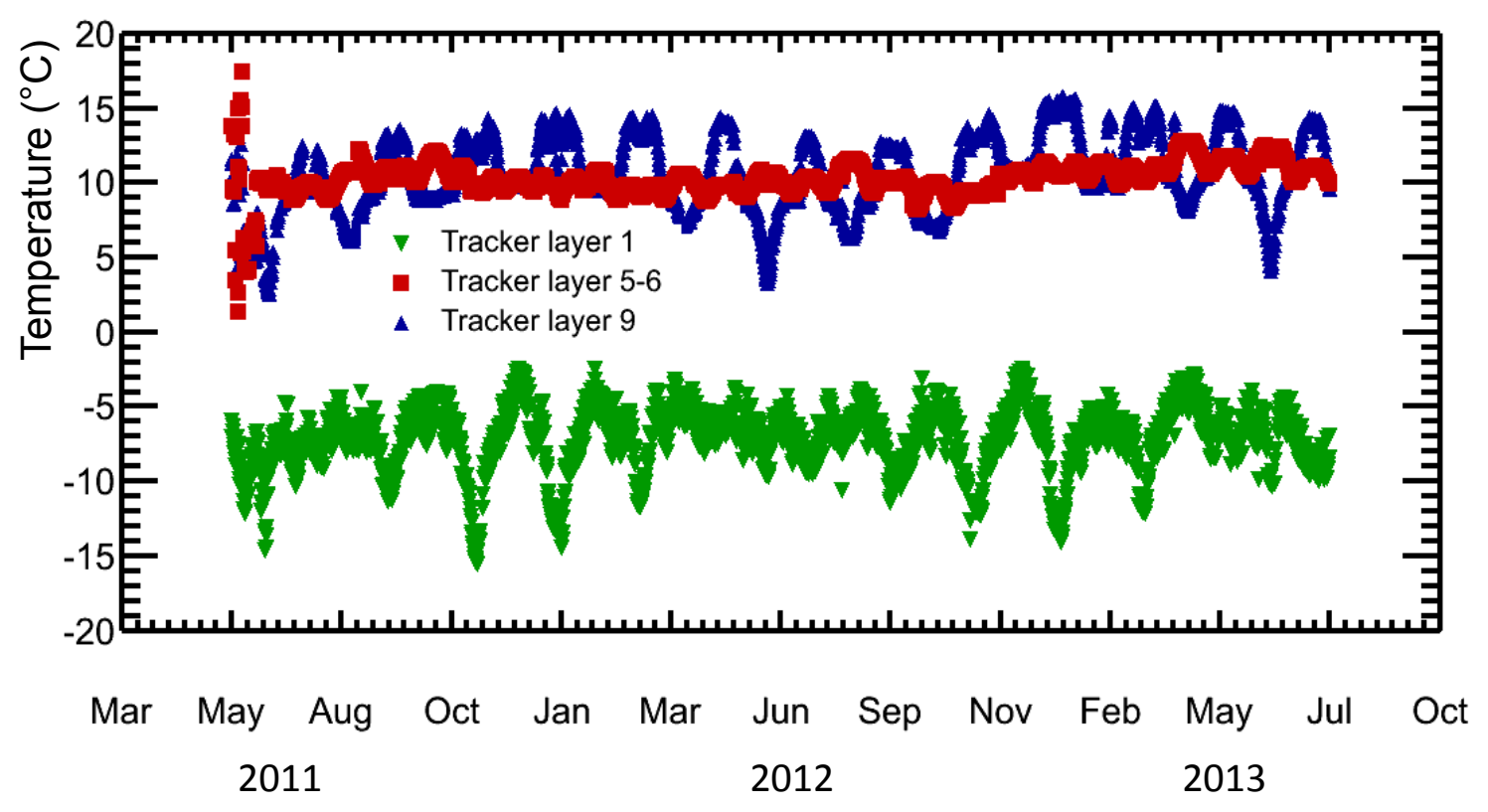

Figure 3: Time evolution of the temperature measured in different sensors thermally connected to the TFEs of the Inner, Upper and Lower Tracker.

RC semi-Gaussian shaper and a sample-and-hold stage. The 1024 analog amplified signals are then multiplexed by the TFE and sent through $>2 \mathrm{~m}$ long coaxial cables to Analog-to-Digital Converters (ADCs). ADCs are located on the Tracker Data Reduction board (TDR) where digitized signals are processed by a Digital Signal Processor (DSP) programmed to perform on-line calibration or data reduction procedures [11].

Ladders are arranged over low density aluminum honeycomb planes, see (c) of Figure 2. The Inner Tracker is constituted by 4 planes. The first plane on top is equipped with ladders on only one side, while the 3 planes inside the magnet have ladders on both sides. The design of the Inner Tracker minimize the amount of material traversed, of the order of $1 \%$ of radiation length, to optimize the momentum measurement at low rigidities. Steps of the Inner Tracker construction and integration in AMS-02 are presented in (d) and (e) of Figure 2. The Tracker is completed by a layer of ladders on top of the TRD (Upper Tracker), and by another layer on the bottom of AMS-02 (Lower Tracker) whose geometry covers the ECAL. Inner, Lower and Upper Tracker are shown in Figure 1).

Details about Tracker ladders construction and testing on-ground can be found in [11] [12].

\section{The Tracker Thermal Control System}

The AMS-02 Tracker Thermal Control System (TTCS) is a mechanically pumped two-phase $\mathrm{CO}_{2}$ cooling loop. It provides an accurate temperature control of the Tracker FTE, removing 140 $\mathrm{W}$ of heat and transporting it to two dedicated radiators facing outer space located on the top of AMS-02. TTCS provides also stability with respect to the environmental temperature changes due 


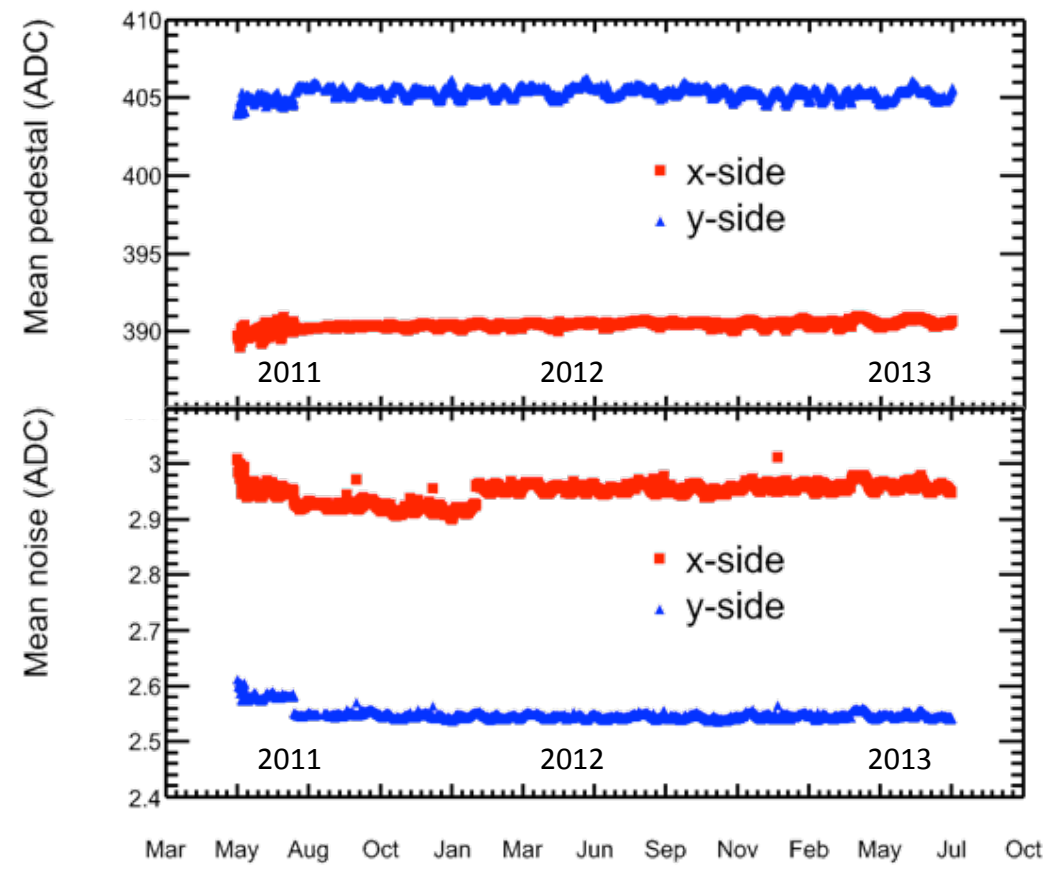

Figure 4: Time evolution of the mean pedestal by side $(x, y)$ and of the average noise. Bad calibrations are not included.

to daily and seasonal Sun exposure variation or due to changes of the attitude of the ISS, as in the case of berthing/unberthing of visiting vehicles [13].

In Figure 3 is presented the average temperature of the Inner, Upper and Lower Tracker TFE during the two years of operation. Since the Lower and Inner Tracker are cooled by the TTCS system, their temperature has a small variation with respect to the orbital thermal environment changes. The wide temperature variations that can be observed at the beginning of AMS-02 operations in 2011 were due to the commissioning of the TTCS system on orbit.

The Upper Tracker instead is facing outer space and has no need of a cooling system. A system of heaters has been installed on this layer to warm up in the case of too low temperatures.

\section{Tracker Data Processing}

The calibration procedure consists in the determination of the pedestal and noise of each of the 196608 readout channels. The procedure is performed every equatorial crossing, corresponding to a period of 46 minutes and lasts for 30 seconds. On equatorial latitudes the particle rate is minimum and the effect of events crossing the detector during calibration is minimized. In Figure 4 the averaged pedestal and noise values are displayed separately for the two sides. The global calibration behavior is stable, and the small changes are due to changes in the calibration configuration or in the electronics [14].

Calibration is then used for the zero-suppression algorithm during the data event processing. First pedestal and the common noise, calculated for each VA, are subtracted from the signal. Then signals exceeding a fix signal-to-noise ratio $(\mathrm{S} / \mathrm{N})$, from 3.5 to 4.0 depending on the TDR con- 

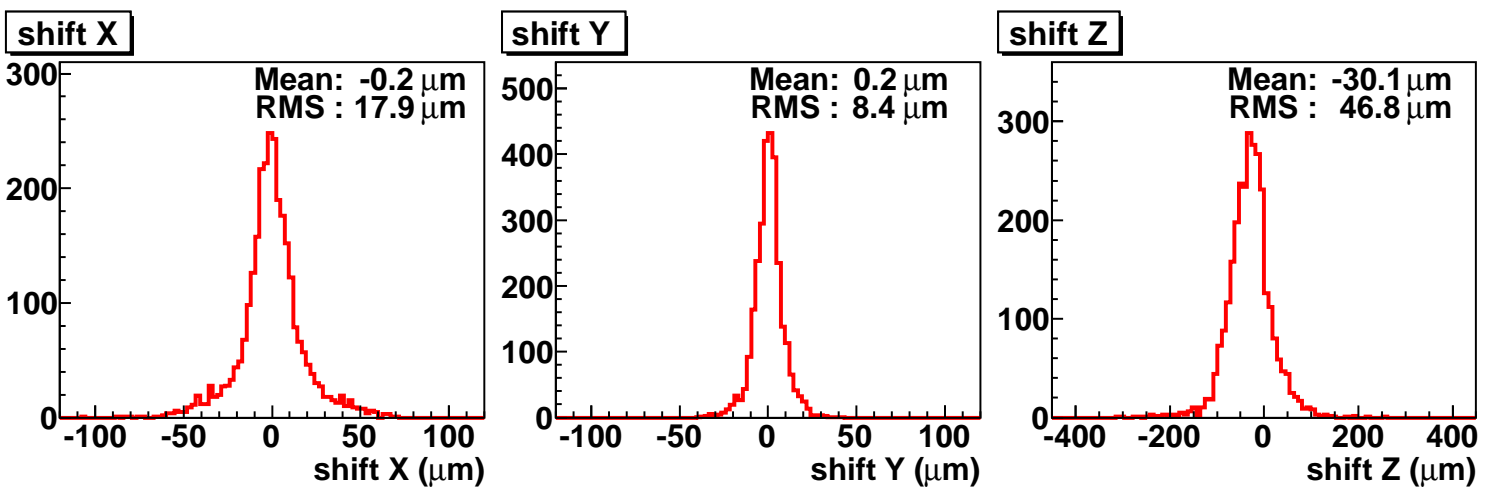

Figure 5: Relative shifts of sensors between test beam and ISS data. The shift in $z$-coordinate is defined with respect to the support frame. The shift of $30 \mu \mathrm{m}$ in $z$-coordinate can be interpreted as out-gassing of the foam support in space.

figuration, trigger the acquisition of the cluster, a collection of neighboring strips that have $\mathrm{S} / \mathrm{N}$ more than 1. The best triggering $\mathrm{S} / \mathrm{N}$ thresholds are determined by a compromise between the efficiency in detecting the crossing particle (a typical signal S/N for a MIP is 5), and the number of noise-generated clusters that should be kept low in order to respect the constraint on the AMS-02 event size due to the limited ISS transmission bandwidth. With the current configuration a typical Tracker event size of $1 \mathrm{~kb} /$ event has been obtained, corresponding to a compression factor of approximately 1000 , keeping a ladder intrinsic efficiency of more than $95 \%$ for more than $90 \%$ of the modules [15].

Data sent on ground are then processed by an offline procedure. First clusters along the $x$ coordinate and the ones along the $y$ coordinate are coupled into three-dimensional hits. All the possible combinations of $x$ and $y$ clusters belonging to the same ladder are created. Then the energy deposition estimation measured independently by the $x$ and the $y$ side are compared, and hits that have a poor agreement are discarded. To achieve the maximum hit reconstruction efficiency on the bending coordinate, hits with only the $y$ information are also created. Hits are then combined to create the track. A brute force algorithm that explores most of all the possible combination is used. Details about the pattern finding procedure and Tracker reconstruction can be found in [4].

The coordinate of passage of the particle is estimated by the center of gravity of the signals of the two higher signal strips. When the inclination of the incoming particle is larger than $20^{\circ}(\mathrm{Y})$ or $35^{\circ}(\mathrm{X})$, the best resolution is obtained using three strips [12]. The resolution are estimated to be 10 (30) $\mu \mathrm{m}$ for vertical $Z=1$ particles on the $y(x)$ coordinate [12]. The resolution improves for $Z>1$ particles up to $5 \mu \mathrm{m}$ giving an higher MDR for helium and higher charge nuclei [4].

\section{Tracker Alignment}

Rigidity measurement and charge sign determination require a knowledge of the relative position of silicon sensors with a precision better than $10 \mu \mathrm{m}$ at any moment. Since the typical Tracker mechanical assembly accuracy was of the order of $100 \mu \mathrm{m}$, an alignment procedure based on test beam data and cosmic-rays on ground was developed [12]. The alignment procedure has been re- 


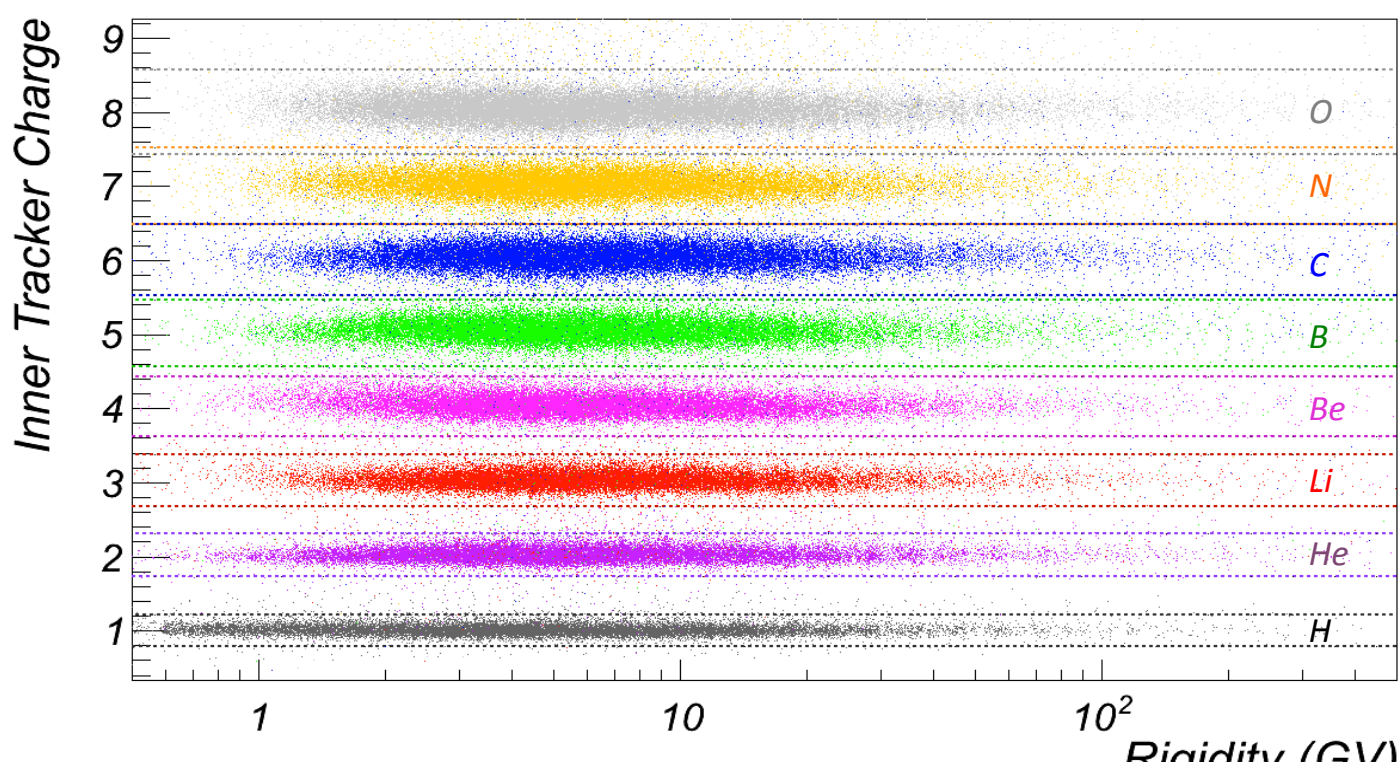

Figure 6: The combined charge measurement of the Inner Tracker layers after all corrections. The dashed lines correspond to a a high efficiency, low contamination 4-sigma selection.

peated in space to correct for the effect of the vibration during the space shuttle launch. Figure 5 shows the relative shifts of sensors between alignment on ground and in space.

The mean shift of $30 \mu \mathrm{m}$ in $z$-coordinate with respect to the support frame is interpreted as the effect of the deformation of the supporting structures of the ladders. In fact the foam that connects the ladder to his rigid carbon and aluminum structure shrinks as an effect of the outgassing in the open space. The same effect was also observed for the AMS-01 silicon Tracker ladders, whose design and materials are similar to the ones adopted for AMS-02 ladders [17].

Inner Tracker planes are held stable by a cylindrical carbon fiber structure mechanically connected with the magnet. Consequently their position does not change with time. Possible movements of these layers are monitored at the few $\mu \mathrm{m}$ level by 20 infrared laser beams traversing the Inner Tracker in the central region [18].

Due to temperature variations along the ISS orbit, displacements of the outer layers were observed at a time scale of tens of minutes, which is due to the thermal deformation of AMS-02 support structure. A dynamic alignment of outer layers with respect to the Inner Tracker have been developed to correct for this effect. The main characteristic of this method is the use of local data around a given moment in time to build the estimate of the position and orientation of outer Tracker layers [16].

The final alignment accuracy has been estimated being $7 \mu \mathrm{m}$ [16].

\section{Tracker Charge Measurement}

The total signal of the cluster is related to energy deposited by ionization in the silicon. To precisely derive the energy deposited in silicon a series of corrections due to detector effects should 


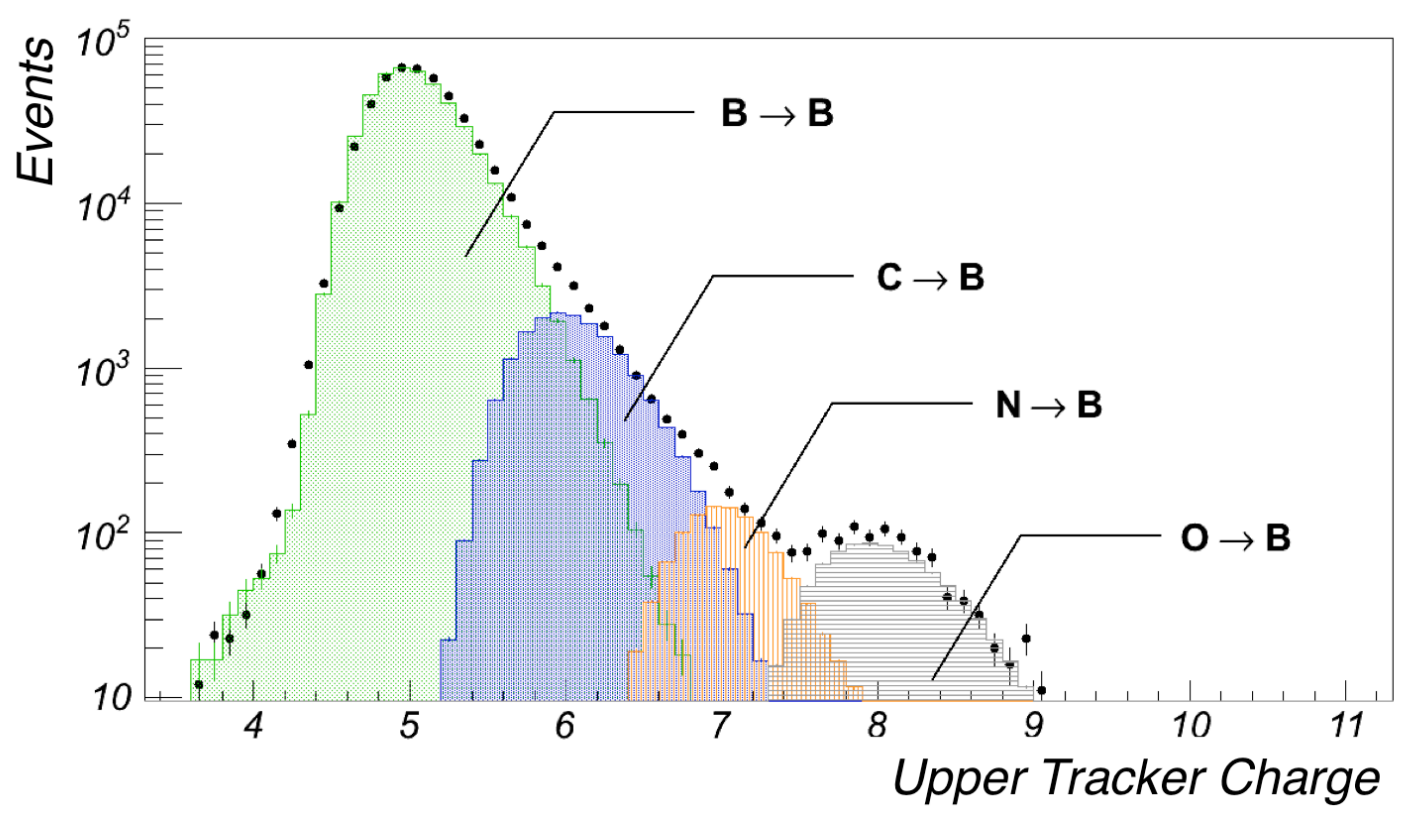

Figure 7: Charge distribution of Upper Tracker for a selection of Boron with TOF and Inner Tracker (filled circles). Number of interacting primaries such as Carbons, Nitrogens and Oxygens can be estimated by a reference spectrum fit.

be applied to the total signal[10]. First the cross-talk between neighboring readout channels is subtracted. Then signals are corrected for the gain factor measured for each VAs. A correction for the cluster total signal dependence on the impact position between strips is then applied. The signal collection efficiency is in fact higher when the particle passes close to a readout strip, while is significantly lower if the impact position is in between the two strips. An additional dependence on the impact angle has been also observed and parametrized. Finally a global linearization of the signal behavior with respect to energy loss has been also introduced.

Energy loss is related to the particle charge via the Bethe-Bloch formula $\mathrm{d} E / \mathrm{d} x=Z^{2} \times f(\beta \gamma)$. To derive an estimation of the charge of the particle $Z$ the energy loss should be divided by the particle path-lenght in the silicon and by its dependence with $\beta \gamma$. An effective description of the Bethe-Bloch energy dependence has been derived using estimators of $\beta \gamma$ from TOF, at low energies, and Tracker, at high energy. Effects of the slow-down due to energy losses in AMS-02 materials have also been included [10].

Finally up to 18 charge measurements can be derived for a single particle passing through the Tracker. These measurements are then combined together in an unique estimator reaching a resolution at the level of 0.1 charge units (c.u.) for Carbon and lower than 0.3 c.u. up to silicon. Charges up to $Z=28$ are detected. An example of the estimator performance, constructed with charge information of the Inner Tracker only, is presented in Figure 6. The dashed lines correspond to a high efficiency, low contamination 4-sigma selection.

\section{Chemical Composition with the Tracker}

The chemical composition of the CRs gives valuable information about CRs life, from the 
sources most probable location, to details about the diffusion in the Galaxy. Among the CRs nuclei measurements the Boron-to-Carbon fluxes ratio is one of the most sensitive observables for the propagation modeling, Boron being a secondary product of spallation of heavier primary elements present in cosmic rays such as Carbon and Oxygen [19].

Separation of Boron and Carbon samples is obtained by means of simple cuts on the Inner Tracker charge estimators, presented in Figure 6. The selection efficiency is $99 \%$ and the misidentification probability is at the level of few permille. The selection is improved using charge estimators coming from the TOF with a global efficiency of $98 \%$ and mis-identification probabilities at the level of $10^{-4}$ [19].

Selection with TOF and Tracker, although very precise, can be biased. In fact incoming nuclei may interact on the upper part of AMS-02 and create secondaries of high energy and lower charge. These events can be detected by the comparison of the Inner Tracker and the Upper Tracker charge measurement. In Figure 7 is presented the charge distribution of the Upper Tracker for a Boron sample selected with the Inner Tracker. A large population of non-interacting Boron can be observed as well as the populations for charge-changing processes $\mathrm{O} \rightarrow \mathrm{B}, \mathrm{N} \rightarrow \mathrm{B}$ and $\mathrm{C} \rightarrow \mathrm{B}$. The interaction can be eliminated by simple cuts on the charge of the Upper Tracker and residual contaminations estimated by a reference spectrum fit [19].

\section{Conclusion}

In more than two years of continuos operation in space the AMS-02 silicon Tracker and the Tracker Thermal Control System have been operating successfully. Procedures for the sensors alignment and VA signal response equalization were developed. Overall performances in terms of tracking efficiency, rigidity measurement, charge sign determination, and absolute charge magnitude have been obtained. Eventually an example of the use of the Tracker charge magnitude measurement capabilities has been presented in the context of the CRs nuclei physics, showing how the precise and repeated determinations of charge in the AMS-02 Tracker leads to the detection of the interacting and non-interacting events.

\section{References}

[1] J. Alcaraz et al., Phys. Rep. 366 (2002) 331-405.

[2] A. Kounine et al., Int. J. Mod. Phys. E 21 (2012) 1230005.

[3] K. Luebelsmeyer et al., Nucl. Instrum. Methods A 654 (2011) 639.

[4] P. Zuccon et al., Proc. of 33rd ICRC 1064 (2013).

[5] Q. Yan et al., Proc. of 33rd ICRC 1097 (2013).

[6] M. Aguilar et al., Phys. Rev. Lett. 110 (2013) 141102.

[7] F. Giovacchini et al., Proc. of 33rd ICRC 1028 (2013).

[8] W. Sun et al., Proc. of 33rd ICRC 940 (2013).

[9] L. Basara et al., Proc. of 33rd ICRC 917 (2013).

[10] P. Saouter et al., Proc. of 33rd ICRC 789 (2013). 
[11] W. J. Burger et al., Nucl. Instrum. Methods A 512 (2003) 517.

[12] B. Alpat et al., Nucl. Instrum. Methods A 613 (2010) 207.

[13] J. van Es, Proc. of IAC C2.7.1 (2009).

[14] J. L. Bazo Alba et al., Proc. of 33rd ICRC 849 (2013).

[15] J. L. Bazo Alba et al., Proc. of 23rd ECRS, published on JPCS 409 (2012) 012032.

[16] C. Delgado et al., Proc. of 33rd ICRC 1260 (2013).

[17] W. J. Burger et al., Nucl. Instrum. Methods A 512 (2003) 517.

[18] S. Natale et al., Proc. of 31th ICRC 245 (2009).

[19] A. Oliva et al., Proc. of 33rd ICRC 1266 (2013). 